Draft Version SEPtember 18, 2020

Typeset using IATEX default style in AASTeX63

\title{
Detection of a Disk Surrounding the Variably Accreting Young Star HBC722
}

\author{
Xi Yek (Zach),${ }^{1, *}$ Michael M. Dunham,${ }^{1,2, \dagger}$ Héctor G. Arce,${ }^{3}$ Tyler L. Bourke, ${ }^{4}$ Xuepeng Chen,${ }^{5}$ \\ Joel D. Green, ${ }^{6}$ Ágnes Kóspál, ${ }^{7,8,9}$ And Steven N. Longmore ${ }^{10}$ \\ ${ }^{1}$ Department of Physics, State University of New York at Fredonia, 280 Central Ave, Fredonia, NY 14063, USA \\ ${ }^{2}$ Center for Astrophysics| Harvard 83 Smithsonian, 60 Garden Street, Cambridge, MA 02138, USA \\ ${ }^{3}$ Department of Astronomy, Yale University, P.O. Box 208101, New Haven, CT 06520-8101, USA \\ ${ }^{4}$ SKA Organisation, Jodrell Bank, Lower Withington, Macclesfield, Cheshire SK11 9FT, UK \\ ${ }^{5}$ Purple Mountain Observatory, Chinese Academy of Sciences, 2 West Beijing Road, Nanjing 210008, China \\ ${ }^{6}$ Space Telescope Science Institute, 3700 San Martin Dr., Baltimore, MD 02138, USA \\ ${ }^{7}$ Konkoly Observatory, Research Centre for Astronomy and Earth Sciences, Konkoly-Thege Miklós út 15-17, 1121 Budapest, Hungary \\ ${ }^{8}$ Max Planck Institute for Astronomy, Königstuhl 17, 69117 Heidelberg, Germany \\ ${ }^{9}$ ELTE Eötvös Loránd University, Institute of Physics, Pázmány Péter sétány 1/A, 1117 Budapest, Hungary \\ ${ }^{10}$ Astrophysics Research Institute, Liverpool John Moores University, 146 Brownlow Hill, Liverpool L3 5RF, UK
}

Submitted to Research Notes of the AAS

\begin{abstract}
We present new ALMA $233 \mathrm{GHz}$ continuum observations of the FU Orionis Object HBC722. With these data we detect HBC722 at millimeter wavelengths for the first time, use this detection to calculate a circumstellar disk mass of $0.024 \mathrm{M}_{\odot}$, and discuss implications for the burst triggering mechanism.
\end{abstract}

\section{INTRODUCTION}

FU Orionis objects (hereafter FUors) are a group of pre-main-sequence stars that abruptly increase in brightness by several magnitudes, lasting decades or longer. There are about $10-20$ FUors confirmed by direct observation of their bursts, along with an approximately equal number of candidates found to display similar spectral characteristics (e.g., Reipurth \& Aspin 2010). The FUors large amplitude bursts are credited to enhanced mass accretion from the surrounding circumstellar disk, with various triggering mechanisms being proposed over the years, e.g. gravitational and/or magnetorotational instabilities, thermal instabilities, or interactions with binary companions (e.g., Hartmann \& Kenyon 1985, 1996; Audard et al. 2014). FUors may represent the late stages of a cycle of episodic accretion bursts and luminosity flares during the embedded phase (Dunham et al. 2014b). Thus, studying and characterizing each FUor is important for understanding how they fit into the general star formation process.

In this paper, we present new $1 \mathrm{~mm}$ continuum observations of the FUor HBC722 obtained with the Atacama Large Millimeter/Submillimeter Array (ALMA). HBC722, located in the North American/Pelican Nebula Complex $(d \sim 800 \mathrm{pc}$; Zucker et al. 2020), was undetected in previous $1 \mathrm{~mm}$ continuum data from the Submillimeter Array (SMA), setting an upper limit for the disk mass of $0.05 \mathrm{M}_{\odot}$ (after rescaling to the current distance; Dunham et al. 2012). With our new ALMA data we present the first millimeter continuum detection of this object, and use our data to calculate the mass of its circumstellar disk.

\section{OBSERVATIONS \& RESULTS}

We obtained ALMA Cycle 2, Band 6, 12-m array observations of HBC722 on 2014 May 01 and 2015 May 03, with $34-36$ operational antennas. The array configuration provided projected baseline lengths of $12-450 \mathrm{~m}$ and a synthesized beam size (assuming natural weighting) of $1.67 " \times 0.89 "$ at a position angle of $5.82^{\circ}$ (measured east of north). To measure the continuum with the widest possible bandwidth while avoiding contamination from bright lines, the four spectral windows were centered at 224, 226, 240, and $242 \mathrm{GHz}$, and were configured to provide 128 channels over $1.875 \mathrm{GHz}$ bandwidth. The resulting continuum image is centered at $233 \mathrm{GHz}(1.29 \mathrm{~mm})$ and has a total bandwidth of $7.5 \mathrm{GHz}$.

*xyek@fredonia.edu

$\dagger$ michael.dunham@fredonia.edu 


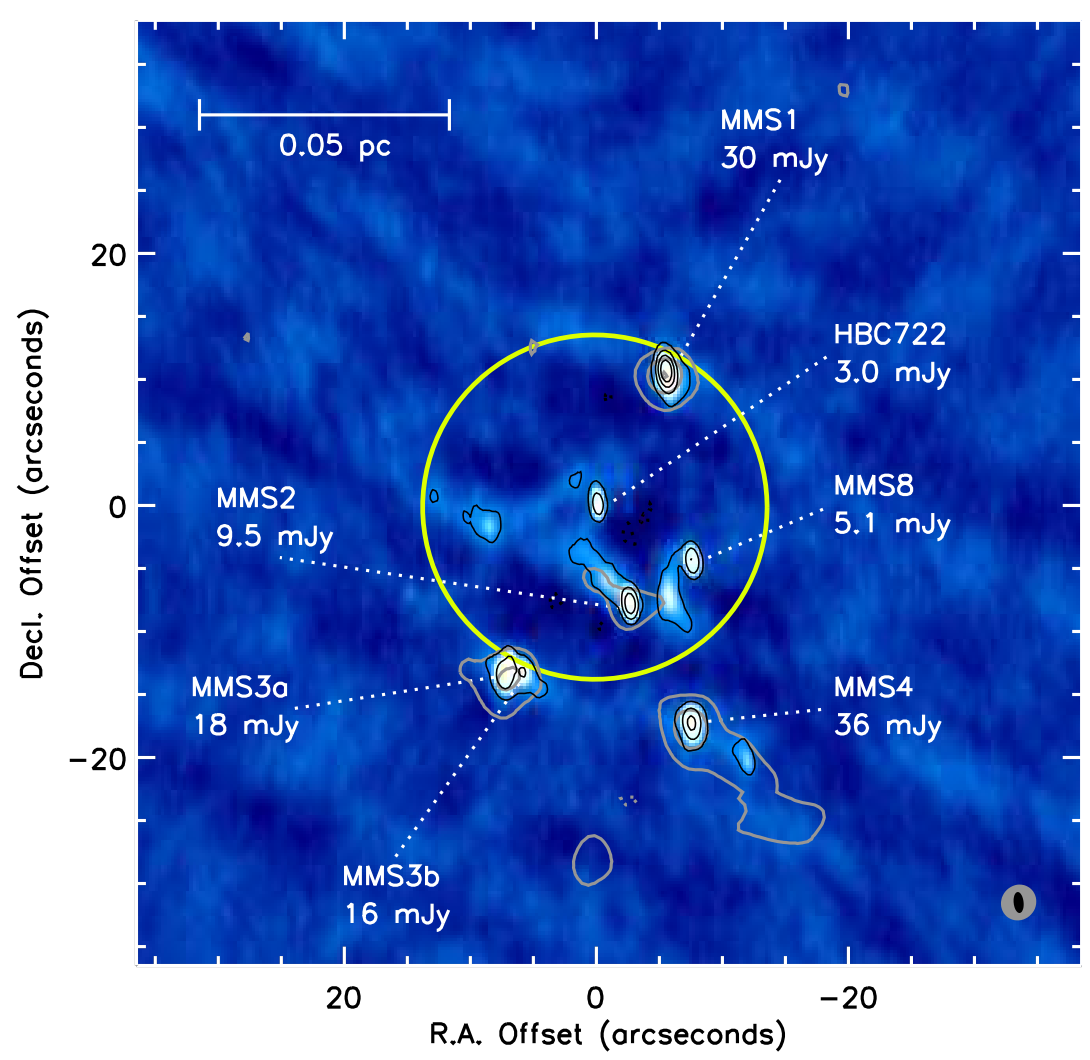

Figure 1. ALMA $233 \mathrm{GHz}$ continuum image of HBC722. The yellow circle shows the ALMA primary beam. The black contours show the ALMA continuum data at levels of 5, 20, 50, and 100 times the $1 \sigma$ rms noise, whereas the gray contours show the SMA continuum data at approximately the same frequency from Dunham et al. (2012), starting at $3 \sigma$ and increasing in steps of $5 \sigma$. The ALMA and SMA synthesized beams are shown as the black and gray ellipses, respectively. Detections from Dunham et al. (2012) are labeled, with annotations reporting their integrated flux densities from the primary beam corrected ALMA data. MMS3 from Dunham et al. (2012) is resolved as two sources in our higher-resolution ALMA data, MMS5, MMS6, and MMS7 from Dunham et al. (2012) are too far outside the ALMA primary beam to be robustly detected, and MMS8 is a new ALMA detection that was only marginally detected in the SMA data.

Calibration was performed using the Common Astronomy Software Applications (CASA) package ${ }^{1}$, following the standard techniques described in Petry et al. (2014) and Schnee et al. (2014). We then applied two rounds of phase self-calibration, followed by one round of amplitude self-calibration. The effective solutions intervals used for selfcalibration were $390 \mathrm{~s}$ (first round of phase calibration), $30 \mathrm{~s}$ (second round of phase calibration), and $900 \mathrm{~s}$ (amplitude calibration). After applying the amplitude self-calibration we verified that the noise in the image decreased while the total fluxes of the detected objects did not change. The self-calibrated image has a $1 \sigma \mathrm{rms}$ of $0.057 \mathrm{mJy}_{\mathrm{beam}}{ }^{-1}$.

In Figure 1, which displays the self-calibrated ALMA $233 \mathrm{GHz}$ continuum image, HBC722 is detected with a peak signal-to-noise ratio of 51 . With a peak intensity of $2.9 \mathrm{mJy}^{\text {beam }}{ }^{-1}$, the ALMA detection of HBC722 is consistent with the previous SMA non-detection from Dunham et al. (2012) at approximately the same frequency.

\section{ANALYSIS \& CONCLUSIONS}

We used the CASA tool GAUssfit to fit an elliptical Gaussian in the image plane to all of the detected sources in the ALMA continuum image. The resulting integrated flux densities are included as annotations in Figure 1. For

${ }^{1}$ Available at http://casa.nrao.edu 
HBC722, we then calculated the total circumstellar disk mass $M$ as:

$$
M=100 \frac{d^{2} S_{\nu}}{B_{\nu}\left(T_{D}\right) \kappa_{\nu}},
$$

where $d=800 \mathrm{pc}, S_{\nu}$ is the measured integrated flux density, $B_{\nu}\left(T_{D}\right)$ is the Planck function at the isothermal dust temperature $T_{D}, \kappa_{\nu}$ is the opacity of the dust, and the factor of 100 is the assumed gas-to-dust ratio. We adopt the dust opacities of Ossenkopf \& Henning (1994) for thin ice mantles after $10^{5}$ yrs of coagulation at a gas density of $10^{6} \mathrm{~cm}^{-3}$, giving $\kappa_{\nu}=0.901 \mathrm{~cm}^{2} \mathrm{~g}^{-1}$ at $233 \mathrm{GHz}$. Assuming $T_{D}=30 \mathrm{~K}$, Equation 1 gives a total circumstellar disk mass for HBC722 of $0.024 \mathrm{M}_{\odot}$. With typical uncertainties of a factor of a few (Dunham et al. 2014a), this result is in good agreement with the total gas mass of $0.03 \mathrm{M}_{\odot}$ found by Kóspál et al. (2016).

With a burst accretion rate of $10^{-6} \mathrm{M}_{\odot} \mathrm{yr}^{-1}$ (Kóspál et al. 2011), it would take 24,000 yr to drain a $0.024 \mathrm{M}_{\odot}$ disk. Thus this disk mass is sufficient to power the current accretion burst. With a known stellar mass of approximately $0.5 \mathrm{M}_{\odot}$ (Cohen \& Kuhi 1979), our calculated disk mass implies that HBC722 has a disk-to-star mass ratio of approximately $5 \%$. While this is a factor of ten higher than the median disk-to-star mass ratio for $\mathrm{T}$ Tauri stars (Andrews \& Williams 2005), it is still marginally too low for gravitational instabilities to serve as the burst triggering mechanism, as such instabilities likely require disk-to-star mass ratios of $10 \%$ or higher. However, given the uncertainties in the calculated mass, and the possibility that the ALMA continuum detection is optically thick, gravitational instabilities remain a possible triggering mechanism for this object. Future analysis using radiative transfer modeling is required to better determine the true mass of the HBC722 disk.

\section{ACKNOWLEDGMENTS}

This paper makes use of the following ALMA data: ADS/JAO.ALMA\#2013.1.00088.S. ALMA is a partnership of ESO (representing its member states), NSF (USA) and NINS (Japan), together with NRC (Canada), MOST and ASIAA (Taiwan), and KASI (Republic of Korea), in cooperation with the Republic of Chile. The Joint ALMA Observatory is operated by ESO, AUI/NRAO and NAOJ. The National Radio Astronomy Observatory is a facility of the National Science Foundation operated under cooperative agreement by Associated Universities, Inc. This project has received funding from the European Research Council (ERC) under the European Union's Horizon 2020 research and innovation programme under grant agreement No 716155 (SACCRED). This research has made use of NASA's Astrophysics Data System (ADS) Abstract Service and the IDL Astronomy Library hosted by the NASA Goddard Space Flight Center.

\section{REFERENCES}

Andrews, S. M., \& Williams, J. P. 2005, ApJ, 631, 1134, doi: $10.1086 / 432712$

Audard, M., Ábrahám, P., Dunham, M. M., et al. 2014, in Protostars and Planets VI, ed. H. Beuther, R. S. Klessen, C. P. Dullemond, \& T. Henning, 387

Cohen, M., \& Kuhi, L. V. 1979, ApJS, 41, 743, doi: 10.1086/190641

Dunham, M. M., Arce, H. G., Bourke, T. L., et al. 2012, ApJ, 755, 157, doi: 10.1088/0004-637X/755/2/157

Dunham, M. M., Vorobyov, E. I., \& Arce, H. G. 2014a, MNRAS, 444, 887, doi: 10.1093/mnras/stu1511

Dunham, M. M., Stutz, A. M., Allen, L. E., et al. 2014b, in Protostars and Planets VI, ed. H. Beuther, R. S. Klessen, C. P. Dullemond, \& T. Henning, 195

Hartmann, L., \& Kenyon, S. J. 1985, ApJ, 299, 462, doi: $10.1086 / 163713$
-. 1996, ARA\&A, 34, 207, doi: 10.1146/annurev.astro.34.1.207

Kóspál, Á., Ábrahám, P., Acosta-Pulido, J. A., et al. 2011, A\&A, 527, A133, doi: 10.1051/0004-6361/201016160

-. 2016, A\&A, 596, A52, doi: 10.1051/0004-6361/201528061

Ossenkopf, V., \& Henning, T. 1994, A\&A, 291, 943

Petry, D., Vila-Vilaro, B., Villard, E., Komugi, S., \& Schnee, S. 2014, in Society of Photo-Optical Instrumentation Engineers (SPIE) Conference Series, Vol. 9152, Software and Cyberinfrastructure for Astronomy III, 91520J

Reipurth, B., \& Aspin, C. 2010, in Evolution of Cosmic Objects through their Physical Activity, ed. H. A. Harutyunian, A. M. Mickaelian, \& Y. Terzian, 19-38 
Schnee, S. L., Brogan, C., Espada, D., et al. 2014, in Society of Photo-Optical Instrumentation Engineers (SPIE) Conference Series, Vol. 9149, Observatory Operations: Strategies, Processes, and Systems V, 91490Z
Zucker, C., Speagle, J. S., Schlafly, E. F., et al. 2020, A\&A, 633, A51, doi: 10.1051/0004-6361/201936145 